\title{
Suplementación con hidroxianálogo de metionina: efecto sobre el desempeño productivo en vacas lecheras ${ }^{1}$
}

\section{Supplementation of methionine hydroxy analog: effect on dairy cow performance}

\author{
Omar Vargas-Villalobos ${ }^{2}$, Oscar Cambronero-Castro ${ }^{3}$, Jorge Alberto Elizondo-Salazar ${ }^{4}$
}

\begin{abstract}
Resumen
Suplementar vacas lecheras con dietas bajas en proteína con aminoácidos específicos, es una estrategia promisoria para contrarrestar el potencial efecto negativo de una deficiencia de proteína metabolizable en la productividad. El objetivo del presente estudio fue cuantificar el desempeño productivo de vacas lactantes y la calidad de la leche ante dos dietas con diferente concentración de proteína cruda (PC) y el suministro de hidroxianálogo de metionina (HMTBA). El estudio se realizó en una finca lechera comercial en la localidad de Alfaro Ruiz, Alajuela, Costa Rica, durante el primer semestre del 2014. Veinte vacas multíparas de la raza Holstein bajo un sistema de pastoreo, fueron agrupadas de acuerdo con los días de lactancia y número de parto. Dos dietas con diferentes concentraciones de PC (alto 16,6\% y estándar $15,8 \%)$ con o sin inclusión de HMTBA (25 g/tm) fueron estudiadas en un arreglo factorial 2x2. No se encontraron efectos significativos $(\mathrm{P}>0,05)$ sobre la producción de leche, leche corregida al $4 \%$ de grasa, grasa láctea, proteína láctea, lactosa, sólidos totales o nitrógeno ureico en leche (NUL) al incluir HMTBA. Alimentar las vacas con alta concentración de PC incrementó significativamente $(\mathrm{P}<0,05)$ la concentración de NUL de 18,33 a 20,70 mg/dl. Suplementar HMTBA a vacas en pastoreo no tuvo una respuesta significativa $(\mathrm{P}>0,05)$ en las diferentes variables estudiadas y tampoco se encontró una retribución económica, debido a que no se incrementó la cantidad de leche ni los sólidos totales.
\end{abstract}

Palabras clave: aminoácidos esenciales, análogo de metionina, sólidos lácteos, producción de leche.

\begin{abstract}
Supplementing dairy cows with low in protein diets with specific amino acids is a promising strategy to counteract the potential negative effect of metabolizable protein deficiency on productivity. The objective of this study was to quantify the productive performance of lactating dairy cows and their milk quality when they are offered two diets with different crude protein $(\mathrm{CP})$ concentration and when they are supplemented with methionine hydroxy analog (HMTBA). This study was carried out in a commercial dairy farm located in Alfaro Ruiz-Alajuela, Costa Rica, during the first semester of 2014. Twenty multiparous Holstein cows under a grazing system were grouped according to days in lactation and calving number. Two diets with different concentrations of CP (high $16.6 \%$ and $15.8 \%$ standard), with or without inclusion of HMTBA $(25 \mathrm{~g} / \mathrm{TM})$ were studied in a $2 \times 2$ factorial arrangement. No significant effects $(\mathrm{P}>0.05)$ on milk production, $4 \%$ fat corrected milk, milk fat, milk protein, lactose, total solids or milk urea nitrogen (MUN) were found when including HMTBA. Feeding cows with high CP concentration significantly increased $(\mathrm{P}<0.05)$ MUN concentration from 18.33 to $20.70 \mathrm{mg} / \mathrm{dl}$. Supplementing HMTBA to grazing cows did not have a
\end{abstract}

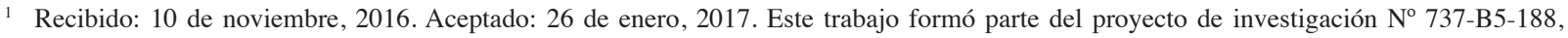
inscrito en la Vicerrectoría de Investigación de la Universidad de Costa Rica (UCR), San José, Costa Rica.

2 Cooperativa de Productores de Leche, Dos Pinos R.L. Alajuela, Costa Rica. omvargas@ dospinos.com

3 Faryvet S.A., Unidad de Investigación. Heredia, Costa Rica. ocambronero@faryvet.com

4 Universidad de Costa Rica, Facultad de Ciencias Agroalimentarias, Estación Experimental Alfredo Volio Mata, Cartago, Costa Rica. jorge. elizondosalazar@ucr.ac.cr (autor para correspondencia). 
significant response $(\mathrm{P}>0.05)$ regarding the different variables, and no financial return was found due to the fact that neither total milk production nor total solids were increased.

Keywords: essential amino acids, methionine analog, milk solids, milk production.

\section{Introducción}

Las vacas en las explotaciones lecheras convierten cerca del 20 al 35\% de la proteína cruda (PC) de la dieta en leche y el resto del nitrógeno $(\mathrm{N})$ lo excretan en las heces y orina, causando problemas ambientales y de manejo de desechos (Elizondo, 2006; Jiménez y Elizondo, 2014). Por esta razón, los productores de leche se encuentran bajo la presión de utilizar sistemas productivos más eficientes y menos contaminantes; se considera que la manera más efectiva de hacerlo es reduciendo la concentración de PC en la dieta. Sin embargo, reducir la concentración de este nutriente y su consumo por debajo de los requerimientos de los animales, conlleva al riesgo de disminuir el consumo de materia seca (MS), y la producción de leche y de proteína láctea (Giallongo et al., 2016).

Suplementar los animales con dietas bajas en PC con aminoácidos (AA) específicos, es una estrategia promisoria para contrarrestar el posible efecto negativo de una deficiencia de proteína metabolizable en la productividad de la vaca lechera (Hristov y Giallongo, 2014).

La metionina (Met) y la lisina (Lis) son considerados los dos aminoácidos más limitantes para la síntesis de leche y la síntesis de proteína láctea (Patterson y Kung, 1988; Piepenbrink et al., 1996; Xu et al., 1998; Chen et al., 2011), ya que la concentración de ambos aminoácidos son los que se excretan en mayor proporción por la glándula mamaria (Bremmer et al., 1997). Además, la lisina se ha identificado como el primer aminoácido limitante cuando la dieta se compone de alimentos a base de maíz y sus derivados, mientras que la metionina se considera limitante cuando las fuentes de proteína de sobrepaso se componen de productos derivados de la soya o proteínas de origen animal (NRC, 2001; Chen et al., 2011).

Existen en el mercado diversos tipos de aminoácidos sintéticos que son comercializados con el objetivo de evitar su degradación a nivel ruminal y lograr así, un impacto a nivel intestinal. Uno de los aminoácidos sintéticos más estudiados en rumiantes es el D-L isómero ácido 2-hidroxi 1-4-metiltio butanóico (HMTBA), el cual posee la característica de no contribuir con $\mathrm{N}$ para suplir el requerimiento nutricional del animal (Wittcoff et al., 2013).

El HMTBA contiene $84 \%$ de metionina, una vez adicionada e ingerida el $60 \%$ será utilizado en el rumen, donde estimulará la síntesis de proteína microbial y el $40 \%$ restante abandonará el rumen con la fase líquida de la ingesta, para ser absorbido a nivel intestinal (Klangnok et al., 2011).

El resultado más consistente al utilizar HMTBA ha sido el incremento en el contenido de grasa láctea (Zanton et al., 2014), y diversos estudios han demostrado también que la suplementación de metionina mejora el perfil de aminoácidos esenciales, lo que es fundamental para maximizar el desempeño en la lactancia y disminuir la excreción de N (Leonardi et al., 2003; Chen et al., 2011), de esta manera se reducen los costos de alimentación y la contaminación ambiental.

El objetivo del presente estudio fue cuantificar el desempeño productivo de vacas lactantes en pastoreo y la calidad de la leche ante dos dietas con diferentes concentraciones de proteína cruda y el suministro de hidroxianálogo de metionina.

\section{Materiales y métodos}

El presente trabajo fue realizado en una explotación lechera comercial en el primer semestre del 2014, ubicada en la localidad de Palmira, Alfaro Ruiz de Alajuela, Costa Rica (10¹2’21,44”N, 84²2’56,67’O). La investigación 
evaluó el desempeño productivo y composición láctea, así como la utilización de nitrógeno ureico en leche (NUL) en vacas lactantes en pastoreo, ante el suministro del hidroxianálogo de metionina (ácido 2 hidroxi-4-metil-tiobutanoico; HMTBA) y dos concentraciones de proteína cruda (PC) dietética.

\section{Composición de las dietas}

Los tratamientos asignados en un arreglo factorial $2 \times 2$, estuvieron conformados por dos concentraciones de PC en una ración parcial mezclada que se ofreció a los animales (16,6\% vs. 15,8\%), con o sin inclusión de HMTBA (25 g/ animal/día). Los cuatro tratamientos fueron: 1) alta concentración de PC 16,6\% más $25 \mathrm{~g}$ de HMTBA (APC+HMTBA), 2) alta concentración de PC 16,6\% sin HMTBA (APC), 3) estándar concentración de PC 15,8\% más 25 g de HMTBA (EPC+HMTBA), y 4) estándar concentración de PC 15,8\% sin HMTBA (EPC). Las dietas fueron balanceadas para vacas que producían en promedio $33,30 \mathrm{~kg}$ de leche y consumían 18,80 kg de materia seca (MS) al día.

Las vacas se encontraban en un sistema de pastoreo rotacional de forraje kikuyo (Kikuyochloa clandestinum) de 32 días de rebrote, y se suplementaban con una ración parcial en canoa, que consistía en pasto kikuyo (Kikuyochloa clandestinum) de corta, cosechado mecánicamente a los noventa días de rebrote, heno de pasto transvala (Digitaria decumbens Stent. cv. Transvala) y suazi (Digitaria swazilandensis), alimento balanceado comercial, pulpa de cítricos deshidratada, harina de soya, grasa de sobrepaso, vitaminas y minerales (Cuadro 1). Esta ración parcial se ofrecía a los animales antes de cada ordeño.

Cuadro 1. Composición química del forraje y de las materias primas utilizadas en la ración parcial mezclada ofrecida a vacas Holstein en la localidad de Alfaro Ruiz de Alajuela, Costa Rica. 2014.

Table 1. Chemical composition of forage and ingredients used in the partial mixed ration fed to Holstein cows, in Alfaro Ruiz, Alajuela, Costa Rica. 2014.

\begin{tabular}{|c|c|c|c|c|c|c|c|}
\hline & $\begin{array}{c}\text { Kikuyo } \\
90 \mathrm{~d}\end{array}$ & $\begin{array}{c}\text { Kikuyo } \\
32 \text { d }\end{array}$ & Heno & $\begin{array}{c}\text { Alimento } \\
\text { balanceado }\end{array}$ & $\begin{array}{c}\text { Pulpa de } \\
\text { cítrico }\end{array}$ & $\begin{array}{c}\text { Harina } \\
\text { soya }\end{array}$ & $\begin{array}{c}\text { Grasa } \\
\text { sobrepaso }\end{array}$ \\
\hline Nutriente & \multicolumn{7}{|c|}{$\mathrm{g} / 100 \mathrm{~g}$ de $\mathrm{MS}$} \\
\hline MS & 18,84 & 18,75 & 86,74 & 87,00 & 87,00 & 89,50 & 88,00 \\
\hline $\mathrm{PC}$ & 15,22 & 25,82 & 4,10 & 16,00 & 4,00 & 48,80 & ----- \\
\hline Cenizas & 13,19 & 10,78 & 12,91 & ----- & ----- & ----- & ----- \\
\hline FDN & 63,20 & 58,15 & 73,83 & 12,00 & 14,00 & 9,80 & ----- \\
\hline FDA & 38,26 & 33,10 & 61,87 & ----- & ----- & 6,20 & ----- \\
\hline \multirow[t]{2}{*}{ Grasa } & ----- & ----- & ----- & ----- & ----- & ----- & 80,88 \\
\hline & \multicolumn{7}{|c|}{ g/100 g de PC } \\
\hline Met & 0,51 & 2,88 & 2,39 & 2,06 & 1,10 & 1,60 & ----- \\
\hline Lis & 0,37 & 1,76 & 2,12 & 2,44 & 0,95 & 2,85 & ----- \\
\hline
\end{tabular}

MS: materia seca, PC: proteína cruda, FDN: fibra en detergente neutro, FDA: fibra en detergente ácido, Met: metionina, Lis: lisina / MS: dry matter, PC: crude protein, FDN: neutral detergent fiber, FDA: acid detergent fiber, Met: methionine, Lis: lysine.

\section{Composición química de la dieta}

Para lograr los tratamientos APC y APC+HMTBA, se adicionó harina de soya en una cantidad que no sobrepasara la concentración de PC recomendada en la ración parcial, y que permitiera observar una diferencia de alrededor de un punto porcentual con respecto a los tratamientos EPC y EPC+HMTBA. La diferencia real entre ambas concentraciones de PC fue de $0,8 \%$. Los tratamientos se formularon de manera que se alcanzara la concentración de proteína degradable en el rumen para no limitar la producción de proteína microbial (Cuadro 2). 
Cuadro 2. Composición química de las dietas utilizadas en vacas Holstein en la localidad de Alfaro Ruiz de Alajuela, Costa Rica. 2014.

Table 2. Chemical composition of the diets fed to Holstein cows, in Alfaro Ruiz-Alajuela, Costa Rica. 2014.

\begin{tabular}{|c|c|c|c|c|}
\hline \multirow[b]{3}{*}{ Ingrediente (\% de la MS) } & \multicolumn{4}{|c|}{ Dietas } \\
\hline & АРC+HMTBA & APC & EPC+HMTBA & EPC \\
\hline & \multicolumn{4}{|c|}{$\mathrm{g} / 100 \mathrm{~g}$ de MS } \\
\hline Kikuyo de 32 días & 24,79 & 24,34 & 28,61 & 27,38 \\
\hline Kikuyo de 90 días & 2,37 & 2,78 & 3,69 & 3,13 \\
\hline Heno & 9,39 & 9,19 & 11,37 & 10,38 \\
\hline Alimento balanceado & 47,71 & 48,71 & 43,17 & 45,40 \\
\hline Harina de soya & 2,86 & 2,81 & 0,36 & 0,26 \\
\hline Pulpa de cítricos deshidratada & 9,83 & 9,64 & 10,79 & 10,80 \\
\hline Grasa de sobrepaso & 1,81 & 1,77 & 1,98 & 2,00 \\
\hline Premezcla vit. y min. & 0,78 & 0,76 & 0,85 & 0,65 \\
\hline HMTBA (84\% Met) & 0,13 & 0,00 & 0,13 & 0,00 \\
\hline \multicolumn{5}{|l|}{ Composición química } \\
\hline MS, g/100 g & 43,59 & 41,96 & 40,51 & 41,53 \\
\hline \multirow[t]{2}{*}{ ENL (Mcal/kg de MS) } & 1,65 & 1,64 & 1,62 & 1,64 \\
\hline & \multicolumn{4}{|c|}{$\mathrm{g} / 100 \mathrm{~g}$ de MS } \\
\hline $\mathrm{PC}$ & 16,60 & 16,60 & 15,80 & 15,80 \\
\hline PDR ( $\%$ de PC) & 70,24 & 68,72 & 71,68 & 71,10 \\
\hline PNDR ( $\%$ de PC) & 29,88 & 31,28 & 28,57 & 28,90 \\
\hline $\mathrm{Ca}+\mathrm{P}$ & 1,66 & 1,66 & 1,72 & 1,62 \\
\hline FDN & 35,46 & 34,84 & 38,08 & 37,06 \\
\hline FDA & 22,28 & 22,04 & 24,40 & 23,60 \\
\hline $\mathrm{CNF}$ & 32,96 & 33,24 & 30,88 & 31,90 \\
\hline \multicolumn{5}{|l|}{ Consumo } \\
\hline MS, kg/día & 19,48 & 20,13 & 17,97 & 17,60 \\
\hline
\end{tabular}

MS: materia seca, PC: proteína cruda, PDR: proteína degradable en el rumen, PNDR: proteína no degradable en el rumen, FDN: fibra detergente neutro, FDA: fibra detergente ácido, CNF: carbohidratos no fibrosos, HMTBA: hidroxianálogo de metionina, APC+HMTBA: dieta alta en proteína cruda con HMTBA, APC: dieta alta en proteína cruda sin HMTBA, EPC+HMTBA: dieta con concentración estándar de proteína con HMTBA, EPC: dieta con concentración estándar de proteína cruda sin HMTBA / MS: dry matter, PC: crude protein, PDR: rumen degradable protein, PNDR: rumen undegradable protein, FDN: neutral detergent fiber, FDA: acid detergent fiber, CNF: non fibrous carbohydrates, HMBTA: methionine hydroxy analog, APC+HMTBA: high crude protein diet with HMTBA, APC: high crude protein diet without HMBTA, EPC+HMTBA: standard crude protein diet with HMTBA, EPC: standard crude protein diet without HMTBA.

\section{Unidad experimental}

El estudio tuvo una duración de cincuenta días, de los cuales quince fueron para adaptación a las dietas y condiciones del experimento, y el resto de días fueron para la evaluación experimental. Se utilizaron de manera simultánea veinte vacas multíparas de la raza Holstein, que promediaron $138( \pm 11)$ días en lactancia, 33,30 $( \pm 3,60) \mathrm{kg}$ de producción de leche y $657,0( \pm 46,00) \mathrm{kg}$ de peso vivo. 


\section{Muestreo y análisis}

Las vacas se ordeñaron dos veces al día (1:00 am y 2:00 pm), la producción láctea de ambos ordeños se registró una vez por semana, se tomaron dos muestras homogéneas de ambos ordeños una vez por semana para cada animal, y se analizaron para composición láctea. Las muestras se analizaron en el laboratorio de recibo de leche de la Cooperativa de Productores de Leche Dos Pinos R.L. para determinar la concentración de PC, grasa, lactosa y NUL.

Los análisis de composición láctea y sólidos totales en leche fueron determinados con base en un análisis con infrarrojo. Para la determinación de NUL se utilizó el método enzimático y la cuantificación por espectrofotometría. La concentración de NUL se analizó en el laboratorio de alimentos balanceados de la misma cooperativa. La cantidad de ración parcial mezclada ofrecida y rechazada se midió una vez por semana, y el forraje consumido mediante pastoreo se determinó semanalmente para todo el grupo de animales, utilizando el método Botanal ${ }^{\circledR}$ (Hargraves y Kerr, 1978). A lo largo del ensayo se tomaron muestras de forraje para determinar su composición nutricional; también se tomaron muestras de los alimentos que aportaban PC para analizar el perfil de AA al inicio del ensayo.

Para los análisis bromatológicos de los forrajes, la concentración de MS, PC y cenizas, se determinaron de acuerdo con la metodología de la AOAC (1990). La concentración de fibra detergente neutro (FDN) y fibra detergente ácido (FDA) se determinó utilizando la metodología descrita por Van-Soest et al. (1991). Para el análisis del perfil de AA se utilizó la técnica cuantitativa cromatográfica HPLC (Bartolomeo y Maisano, 2006).

Se realizó también un análisis económico con el objetivo de determinar el ingreso por concepto de venta de leche, tomando como referencia el costo de las dietas incluyendo el costo del aditivo utilizado. Para determinar el precio de la leche, se tomó en consideración el pago de los componentes lácteos reportados por la Cooperativa de Productores de Leche Dos Pinos R.L el día 4 de junio del año 2014.

\section{Descripción del modelo estadístico}

Se utilizó un diseño de bloques completos al azar con arreglo factorial 2x2. Las vacas se asignaron para cada bloque de acuerdo con el número de parto y días en lactancia. Se utilizaron cinco bloques con cuatro unidades experimentales cada uno. Los datos obtenidos fueron analizados mediante el procedimiento MIXED de SAS (SAS, 2004), donde la producción de leche de la vaca en la lactancia anterior se utilizó como covariable. El efecto del tratamiento se consideró significativo cuando $\mathrm{P} \leq 0,05$ y con tendencia cuando $0,05<\mathrm{P}<0,10$; y se realizó la comparación entre medias por medio de la prueba Waller-Duncan.

\section{Resultados}

\section{Respuesta en la producción láctea}

No hubo interacción entre los diferentes factores y no se encontraron efectos significativos $(\mathrm{P}>0,05)$ sobre la producción de leche, leche corregida al $4 \%$ de grasa, grasa láctea, proteína láctea, lactosa, sólidos totales o NUL al suplementar con HMTBA (Cuadro 3). Sin embargo, al comparar los tratamientos sin el aditivo, es decir considerando solamente la concentración de PC, sí se encontraron diferencias significativas $(\mathrm{P}<0,05)$. Los animales que consumieron la dieta APC tuvieron una producción de leche promedio de 37,60 kg/d, mientras que las vacas en el tratamiento EPC produjeron en promedio $31,01 \mathrm{~kg} / \mathrm{d}$. 
Cuadro 3. Efecto de la dieta sobre la producción de leche y componentes lácteos en vacas Holstein en la localidad de Alfaro Ruiz de Alajuela, Costa Rica. 2014.

Table 3. Effects of the diet on milk production and milk components in Holstein cows, in Alfaro Ruiz-Alajuela, Costa Rica. 2014.

\begin{tabular}{lccccc}
\hline & \multicolumn{3}{c}{ Dietas } \\
\cline { 2 - 6 } & APC+HMTBA & APC & EPC+HMTBA & EPC & DE* \\
\hline Rendimiento leche, kg/día & $35,01 \mathrm{a}$ & $37,60 \mathrm{a}$ & $29,60 \mathrm{~b}$ & $31,01 \mathrm{~b}$ & 1,33 \\
Leche corregida 4\% de grasa (kg/día) & $30,40 \mathrm{a}$ & $32,93 \mathrm{a}$ & $27,43 \mathrm{~b}$ & $26,15 \mathrm{~b}$ & 1,36 \\
Grasa láctea (kg/día) & 1,10 & 1,21 & 1,05 & 1,00 & 0,08 \\
Porcentaje & 3,14 & 3,21 & $3,56 \mathrm{x}$ & $3,21 \mathrm{y}$ & 0,26 \\
Proteína láctea (kg/día) & $1,01 \mathrm{a}$ & $1,12 \mathrm{a}$ & $0,88 \mathrm{~b}$ & $0,89 \mathrm{~b}$ & 0,04 \\
Porcentaje & 2,88 & 2,98 & 2,96 & 2,87 & 0,04 \\
Lactosa (kg/día) & 1,88 & 2,06 & 1,57 & 1,70 & 0,06 \\
Porcentaje & 4,67 & 4,76 & 4,63 & 4,74 & 0,04 \\
Sólidos totales (kg/día) & 3,99 & 4,38 & 3,50 & 3,59 & 0,21 \\
Porcentaje & 11,40 & 11,66 & 11,84 & 11,57 & 0,25 \\
NUL (mg/dl) & $21,10 \mathrm{a}$ & $20,30 \mathrm{a}$ & $17,95 \mathrm{~b}$ & $18,71 \mathrm{~b}$ & 1,09 \\
\hline
\end{tabular}

* DE: desviación estándar, NUL: nitrógeno ureico en leche, HMTBA: hidroxianálogo de metionina, APC+HMTBA: dieta alta en proteína cruda con HMTBA, APC: dieta alta en proteína cruda sin HMTBA, EPC+HMTBA: dieta con concentración estándar de proteína con HMTBA, EPC: dieta con concentración estándar de proteína cruda sin HMTBA / DE: standard deviation, NUL: milk urea nitrogen, HMTBA: methionine hydroxy analog, APC+HMTBA: high crude protein diet with HMTBA, APC: high crude protein diet without HMBTA, EPC+HMTBA: standard crude protein diet with HMTBA, EPC: standard crude protein diet without HMTBA.

${ }^{x y}$ Diferente letra en una misma fila dentro de la misma concentración de PC muestran una tendencia $(0,05<\mathrm{P}<0,10) /$ different letter in the same row within the same $\mathrm{CP}$ concentration show a tendency $(0.05<\mathrm{P}<0.10)$.

${ }^{\text {ab }}$ Diferente letra en una misma fila son diferentes $(\mathrm{P}<0,05) /$ different letter in the same row are different $(\mathrm{P}<0.05)$.

\section{Concentración y producción de grasa láctea}

En cuanto a la producción y concentración de grasa láctea no se encontraron efectos significativos $(\mathrm{P}>0,05)$; sin embargo, se encontró una tendencia positiva $(\mathrm{P}<0,10)$ en la concentración de grasa láctea en el tratamiento EPC+HMTBA con respecto al tratamiento EPC (Cuadro 3). En el primer caso, la concentración de grasa en la leche fue de $3,56 \%$, mientras que en el caso de los animales que consumieron la dieta EPC fue de 3,21\%.

\section{Concentración y producción de proteína láctea}

Los resultados para la concentración de proteína láctea encontrados en el presente experimento no fueron significativos $(\mathrm{P}>0,05)$ para los diferentes tratamientos, y este valor fue en promedio de $2,92 \%$ para los cuatro tratamientos. Sin embargo, la producción fue estadísticamente $(\mathrm{P}<0,05)$ mayor para los tratamientos APC y APC+ HMTBA. 


\section{Adición de HMTBA y excreción de NUL}

Los animales en los tratamientos APC y APC+HMTBA presentaron concentraciones de NUL superiores $(\mathrm{P}<0,05)$ a los animales en los tratamientos EPC y EPC+HMTBA; sin embargo, no se encontraron diferencias significativas $(\mathrm{P}>0,05)$ al comparar los tratamientos APC y APC+HMTBA o EPC y EPC+HMTBA (Cuadro 3). Las concentraciones de NUL oscilaron entre 17,95 y $21,10 \mathrm{mg} / \mathrm{dl}$.

\section{Composición de la dieta}

La composición química de las dietas utilizadas en el ensayo se presenta en el Cuadro 4. Para lograr los tratamientos APC y APC+HMTBA, se adicionó harina de soya en una cantidad que no sobrepasara la concentración

Cuadro 4. Composición química de las dietas utilizadas en vacas Holstein en la localidad de Alfaro Ruiz de Alajuela, Costa Rica. 2014.

Table 4. Chemical composition of the diets fed to Holstein cows in Alfaro Ruiz-Alajuela, Costa Rica. 2014.

\begin{tabular}{|c|c|c|c|c|}
\hline & \multicolumn{4}{|c|}{ Dietas } \\
\hline & APC+HMTBA & APC & ЕРC+HMTBA & EPC \\
\hline Ingrediente (\% de la MS) & \multicolumn{4}{|c|}{$\mathrm{g} / \mathbf{1 0 0} \mathrm{g}$ de MS } \\
\hline Kikuyo de 32 días & 24,79 & 24,34 & 28,61 & 27,38 \\
\hline Kikuyo de 90 días & 2,37 & 2,78 & 3,69 & 3,13 \\
\hline Heno & 9,39 & 9,19 & 11,37 & 10,38 \\
\hline Alimento balanceado & 47,71 & 48,71 & 43,17 & 45,40 \\
\hline Harina de soya & 2,86 & 2,81 & 0,36 & 0,26 \\
\hline Pulpa de cítricos deshidratada & 9,83 & 9,64 & 10,79 & 10,80 \\
\hline Grasa de sobrepaso & 1,81 & 1,77 & 1,98 & 2,00 \\
\hline Premezcla vit. y min. & 0,78 & 0,76 & 0,85 & 0,65 \\
\hline HMTBA (84\% Met) & 0,13 & 0,00 & 0,13 & 0,00 \\
\hline \multicolumn{5}{|l|}{ Composición química } \\
\hline MS, g/100 g & 43,59 & 41,96 & 40,51 & 41,53 \\
\hline \multirow[t]{2}{*}{ ENL (Mcal/kg de MS) } & 1,65 & 1,64 & 1,62 & 1,64 \\
\hline & \multicolumn{4}{|c|}{$\mathrm{g} / 100 \mathrm{~g}$ de $\mathrm{MS}$} \\
\hline $\mathrm{PC}$ & 16,60 & 16,60 & 15,80 & 15,80 \\
\hline PDR (\% de PC) & 70,24 & 68,72 & 71,68 & 71,10 \\
\hline PNDR (\% de PC) & 29,88 & 31,28 & 28,57 & 28,90 \\
\hline $\mathrm{Ca}+\mathrm{P}$ & 1,66 & 1,66 & 1,72 & 1,62 \\
\hline FDN & 35,46 & 34,84 & 38,08 & 37,06 \\
\hline FDA & 22,28 & 22,04 & 24,40 & 23,60 \\
\hline $\mathrm{CNF}$ & 32,96 & 33,24 & 30,88 & 31,90 \\
\hline \multicolumn{5}{|l|}{ Consumo } \\
\hline MS, kg/día & 19,48 & 20,13 & 17,97 & 17,60 \\
\hline
\end{tabular}

MS: materia seca, PC: proteína cruda, PDR: proteína degradable en el rumen, PNDR: proteína no degradable en el rumen, FDN: fibra detergente neutro, FDA: fibra detergente ácido, CNF: carbohidratos no fibrosos, HMTBA: hidroxianálogo de metionina, APC+HMTBA: dieta alta en proteína cruda con HMTBA, APC: dieta alta en proteína cruda sin HMTBA, EPC+HMTBA: dieta con concentración estándar de proteína con HMTBA, EPC: dieta con concentración estándar de proteína cruda sin HMTBA / MS: dry matter, PC: crude protein, PDR: rumen degradable protein, PNDR: rumen undegradable protein, FDN: neutral detergent fiber, FDA: acid detergent fiber, CNF: non fibrous carbohydrates, HMTBA: methionine hydroxy analog, APC+HMTBA: high crude protein diet with HMTBA, APC: high crude protein diet without HMBTA, EPC+HMTBA: standard crude protein diet with HMTBA, EPC: standard crude protein diet without HMTBA. 
de PC recomendada en la ración parcial y que permitiera observar una diferencia de alrededor de un punto porcentual con respecto a los tratamientos EPC y EPC+HMTBA. La diferencia real entre ambas concentraciones de PC fue de $0,8 \%$. Los tratamientos se formularon de manera que se alcanzara la concentración de proteína degradable en el rumen para no limitar la producción de proteína microbial.

El consumo total de MS por parte de los animales varió entre 17,60 y 20,13 kg/día. De acuerdo con el balance nutricional realizado (Cuadro 5), la cantidad de metionina disponible para absorción a nivel intestinal se incrementó en los tratamientos en los que se adicionó HMTBA. La concentración de metionina como porcentaje de proteína metabolizable (PM) aumentó de 2,10\% a 2,55\% en las dietas APC y de 2,19\% a 2,70\% en los tratamientos EPC. El NRC (2001) no reporta ningún requerimiento para aminoácidos expresado en gramos por día, pero recomienda que la lisina y la metionina deben aportar el 7,2\% y 2,4\% de la PM, respectivamente. En este ensayo no se alcanzó dicho aporte para la lisina y los tratamientos sin adición de HMTBA estuvieron por debajo con respecto a la metionina.

Cuadro 5. Aporte de hidroxianálogo de metionina (HMTBA) en el mejoramiento de aminoácidos metabolizables para vacas Holstein en la localidad de Alfaro Ruiz de Alajuela, Costa Rica. 2014.

Table 5. Methionine Hydroxy Analog (HMTBA) contribution in improving metabolizable aminoacids for Holstein cows in Alfaro Ruiz, Alajuela, Costa Rica. 2014.

\begin{tabular}{lcccc}
\hline & \multicolumn{3}{c}{ Dietas } \\
\cline { 2 - 5 } & APC+HMTBA & APC & EPC+HMTBA & EPC \\
\hline Met protegida HMBTA (g/día) & 8,40 & 0 & 8,40 & 0 \\
Met absorbida (g/día) & 42,60 & 43,40 & 37,80 & 38,00 \\
Total Met absorbida (g/día)* & 51,00 & 43,40 & 46,20 & 38,00 \\
Lis metabolizable (\% de la PM) & 5,82 & 5,77 & 5,84 & 5,84 \\
Met metabolizable (\% de la PM) & 2,55 & 2,10 & 2,70 & 2,19 \\
Relación Lis:Met & 2,28 & 2,74 & 2,17 & 2,67 \\
\hline
\end{tabular}

*Se consideró HMTBA $84 \%$ de DL-metionina, el $60 \%$ fue degradable a nivel ruminal y $40 \%$ fue absorbida a nivel intestinal (Klangnok et al., 2011) / HMTBA $84 \%$ of DL-methionine was considered, $60 \%$ was degradable in the rumen and $40 \%$ was absorbed into the small intestine (Klangnok et al., 2011).

PM: proteína metabolizable, APC+HMTBA: dieta alta en proteína cruda con HMTBA, APC: dieta alta en proteína cruda sin HMTBA, EPC+HMTBA: dieta con concentración estándar de proteína con HMTBA, EPC: dieta con concentración estándar de proteína cruda sin HMTBA / PM: metabolizable protein, APC+HMTBA: high crude protein diet with HMTBA, APC: high crude protein diet without HMBTA, EPC+HMTBA: standard crude protein diet with HMTBA, EPC: standard crude protein diet without HMTBA.

La adición de HMTBA incrementó la concentración de Met disponible, y esto hizo que la relación Lis:Met disminuyera de 2,74 a 2,28 para el tratamiento APC y de 2,67 a 2,17 para el tratamiento EPC.

\section{Análisis de factibilidad}

Al realizar el análisis económico, no se encontraron diferencias significativas $(\mathrm{P}>0,05)$ en las variables de respuesta, ni hubo una retribución económica en el ingreso neto o rentabilidad en los tratamientos en los que se adicionó HMTBA (Cuadro 6). 
Cuadro 6. Análisis de costo:beneficio al suplementar vacas Holstein con hidroxianálogo de metionina (HMTBA) en la localidad de Alfaro Ruiz de Alajuela, Costa Rica. 2014.

Table 6. Cost:benefit analysis by supplementing Holstein cows with methionine hydroxy analog (HMTBA) in Alfaro Ruiz, Alajuela, Costa Rica. 2014.

\begin{tabular}{lccccc}
\hline & \multicolumn{5}{c}{ Dieta } \\
\cline { 2 - 6 } & Precio, $\mathbf{\text { /kg }}$ & APC+HMTBA & APC & EPC+HMBTA & EPC \\
\hline Leche, kg & 35,01 & 37,60 & 29,60 & 31,01 & \\
Grasa, \$ & 4,36 & 4,80 & 5,26 & 4,60 & 4,34 \\
Proteína, \$ & 4,36 & 4,40 & 4,90 & 3,82 & 3,88 \\
Otros sólidos, \$ & 3,55 & 6,70 & 7,30 & 5,59 & 6,04 \\
Ingreso bruto, \$/día & & 0,03 & 17,46 & 14,01 & 14,28 \\
Consumo MS, kg & 19,48 & 20,13 & 17,97 & 17,60 & \\
Costo dieta, \$/día & 7,53 & 7,56 & 6,23 & 6,30 & \\
Tratamiento, \$/día & 0,09 & 0,00 & 0,09 & 0,00 & \\
Costo, \$/animal/día & 7,62 & 7,56 & 6,33 & 6,30 & \\
Ingreso neto, \$/día & 8,27 & 9,89 & 7,69 & 7,98 & \\
Rentabilidad, \% & 44,76 & 56,66 & 54,85 & 55,89 & \\
\hline
\end{tabular}

Tipo de cambio según el BCCR/ Exchange rate according to BCCR: 1 US $\$=\varnothing 553,37$.

APC+HMTBA: dieta alta en proteína cruda con HMTBA, APC: dieta alta en proteína cruda sin HMTBA, EPC+HMTBA: dieta con concentración estándar de proteína con HMTBA, EPC: dieta con concentración estándar de proteína cruda sin HMTBA / APC+HMTBA: high crude protein diet with HMTBA, APC: high crude protein diet without HMBTA, EPC+HMTBA: standard crude protein diet with HMTBA, EPC: standard crude protein diet without HMTBA.

\section{Discusión}

Con respecto al desempeño productivo de los animales, los resultados encontrados en la literatura no son consistentes en cuanto a respuestas positivas en producción láctea ante el uso de HMTBA u algún otro tipo de metionina protegida. De acuerdo con Zanton et al. (2014) al realizar un metaanálisis, concluyeron que no existen respuestas significativas entre rendimiento lácteo y la suplementación de metionina; sin embargo, existió una tendencia de incremento cuando las vacas fueron suplementadas con HMTBA. Esa tendencia no se pudo observar en el presente estudio, ya que adicionar HMTBA en ambas concentraciones de PC no incrementó la producción láctea.

En un estudio desarrollado, en el cual se adicionó metionina protegida en dos dietas con diferente concentración de PC (16,1 y 18,8\%), no se cumplió la hipótesis de que al suplementar este aminoácido en dietas deficientes en metionina se incrementa la producción láctea (Leonardi et al., 2003). Por su parte, Phipps et al. (2008) al utilizar HMBi en dietas con dos concentraciones de PC (16,9 y 14,7\%), no encontraron respuesta positiva en la producción láctea y lo asociaron a un faltante de proteína no degradable en el rumen o a algún aminoácido como la lisina, situación que pudo haberse presentado en el presente estudio, ya que la concentración de proteína no degradable en el rumen estuvo alrededor del 30\% (como porcentaje de la PC) en las cuatro dietas utilizadas.

Otra situación que pudo afectar la falta de respuesta a la suplementación, fue la duración del experimento, ya que en estudios similares, el empleo de un cuadrado latino de solamente cuatro semanas de periodo experimental, pudo afectar la falta de respuesta obtenida en el experimento (Phipps et al., 2008). En otro experimento, St-Pierre y Sylvester (2005) obtuvieron un incremento en el rendimiento lácteo atribuible a una respuesta progresiva que alcanzó significancia a partir de la semana once de diecisiete semanas experimentales, razón por la que se puede 
deducir que el período experimental de cinco semanas utilizado en el presente estudio pudo haber sido también insuficiente para observar una respuesta positiva.

Otros trabajos enfocados en medir la producción de leche al utilizar HMTBA en tratamientos isoprotéicos (Johnson-VanWieringen et al., 2007), indicaron un incremento en la producción de leche corregida al 3,5\% de grasa, al suplementar Lis y HMTBA a partir de la semana catorce y diecisiete post parto, lo que indica que la combinación de suplementar ambos AA y somatotropina bovina recombinante resultan en un mayor uso de AA para la producción láctea. Este resultado no solo confirma el efecto progresivo de HMTBA en el tiempo, sino que también hace énfasis en el hecho de que la disponibilidad de lisina puede ser limitante para la producción de leche. Otros autores también reportaron resultados positivos balanceando concentraciones adecuadas de lisina (St-Pierre y Sylvester, 2005; Johnson-VanWieringen et al., 2007; Chen et al., 2011), con valores entre 6,59 y 6,80\% de este AA como porcentaje de la PM. El contenido promedio de lisina en la presente investigación fue de solamente 5,80\%, por lo que, se puede concluir que su disponibilidad en la dieta pudo ser un factor limitante en la producción de leche.

Con respecto a la concentración y producción de grasa láctea, esta variable ha sido la respuesta positiva más constante reportada en la literatura ante la suplementación de HMBTA (Zanton et al., 2014). Esto concuerda con los resultados de otras investigaciones realizadas. Así, por ejemplo Chen et al. (2011) reportaron efectos positivos en el contenido de grasa láctea al suplementar HMBi en la primera semana de lactancia. En otro estudio se determinó un incremento en el contenido de grasa láctea en las primeras cuatro semanas de lactancia (Johnson-VanWieringen et al., 2007). Otros autores demostraron un incremento en el rendimiento $(\mathrm{kg})$ de grasa láctea atribuido al incremento en producción de leche (Phipps et al., 2008). Por su parte, hay estudios donde no se encontraron diferencias significativas en la concentración o en el rendimiento de grasa láctea, debido al corto período de experimentación (35 días), y al bajo potencial genético de los animales (12,5 kg de leche/día) (St-Pierre y Sylvester, 2005; Rulquin et al., 2006; Klangnok et al., 2011). En un metaanálisis se concluyó que la concentración y el rendimiento de grasa láctea fueron significativamente afectados por la suplementación de HMBTA en comparación con otras fuentes de metionina (Zanton et al., 2014).

La suplementación con HMTBA suele tener un efecto en la fermentación ruminal lo que, induce a un incremento en el rendimiento de grasa láctea asociado al incremento de bacterias celulolíticas, esto en raciones que contenían 50\% de concentrado (Hansen et al., 1991). Se supuso que suplementar metionina produce mayores rendimientos de grasa láctea a partir de la colina sintetizada de metionina de forma parcial (Sharma y Erdman, 1988); esto pudo haber ocurrido en el presente experimento con respecto al tratamiento EPC+HMTBA.

Cuando se hace referencia a la concentración y producción de proteína láctea, la suplementación con HMBTA no ha sido constante en incrementarlos (Zanton et al., 2014), situación que se confirma en varias investigaciones realizadas, en las que se mencionan que la inefectividad en aumentar la proteína láctea se debe a la incapacidad de incrementar la concentración plasmática de metionina por parte de los análogos como HMTBA o HMBi, asociado al bajo impacto de la metionina a nivel intestinal (Rulquin et al., 2006; Klangnok et al., 2011; Whelan et al., 2011). Otras de las causas de los resultados negativos al adicionar este aditivo se pueden atribuir a la etapa de la lactancia (Schwab et al., 1992), ya que su efecto positivo se registra, en su mayoría, a inicios de lactancia. En el presente experimento las vacas se encontraron en 138 días de lactancia como promedio (mediana lactancia), por lo que esta situación pudo afectar negativamente la respuesta al suplementar los animales con HMBTA. Varios autores encontraron un incremento en rendimiento y contenido de proteína láctea en la lactancia temprana utilizando HMBi (St-Pierre y Sylvester, 2005; Phipps et al., 2008), situación que se le atribuyó a la baja concentración de metionina con respecto a la PM de las dietas basales $(1,80$ y $1,86 \%$, respectivamente). En el presente estudio, la concentración de metionina en los tratamientos control fue de casi el $80 \%$ del requerimiento de metionina para vacas en la etapa de lactancia, por lo que otro aminoácidos pudo ser limitante.

Otro factor asociado a la respuesta positiva en producción de proteína láctea observada en otros estudios, es la elevada concentración de metionina plasmática al utilizar HMTBi (Rulquin y Delaby, 1997), ya que permite incrementar la síntesis de proteína láctea por la glándula mamaria (Schwab, 1995). 
La suplementación de HMTBA tuvo un efecto significativo sobre la concentración de NUL; sin embargo, no se encontraron diferencias significativas $(\mathrm{P}>0,05)$ al adicionar el aditivo dentro de las mismas concentraciones de PC. Esto es de esperar, ya que los valores de NUL están relacionados con la concentración de PC en la dieta (Jonker et al., 1998).

El actual conocimiento en nutrición de aminoácidos en rumiantes, apoya el concepto que cuando un aminoácido esencial es absorbido como requerimiento del animal, este se reduce y la eficiencia de aminoácidos para la síntesis de proteína se maximiza (NRC, 2001). Adicionar metionina en una dieta deficiente permite mejorar el perfil de aminoácidos absorbidos, resultando en un incremento en la síntesis de proteína láctea por lo que, el N será utilizado para producir proteína láctea en lugar de ser excretado como urea.

Alimentar con HMTBA permitió observar ese concepto en los tratamientos con un EPC al disminuir el valor de NUL, probablemente asociados con la producción de proteína láctea, no así con APC, resultado que es apoyado por St-Pierre y Sylvester (2005) y Chen et al. (2011), quienes al adicionar HMTBi lograron disminuir el valor de NUL y mejorar la eficiencia de utilización de $\mathrm{N}$.

Con respecto al consumo total de MS, no se encontraron diferencias significativas $(\mathrm{P}>0,05)$ para los diferentes tratamientos. Otros estudios tampoco determinaron diferencias en el consumo de MS ante el suministro de HMTBA (Chen et al., 2011; Klangnok et al., 2011; Lapierre et al., 2011). Sin embargo, Polan et al. (1991) encontraron una depresión en el consumo de MS al suministrar grandes cantidades de metionina protegida en la lactancia temprana, asociada a factores organolépticos.

La adición de HMTBA condujo a un incremento en la concentración de metionina disponible, y esto hizo que la relación Lis:Met disminuyera. Lo anterior se puede atribuir al bajo aporte de lisina en las fuentes de alimento utilizadas en este ensayo (Cuadro 1) y al bajo aporte de proteína no degradable en el rumen de las dietas (Cuadro 2). El NRC (2001) indicó que la relación Lis:Met debe de ser de 3,0:1,0.

El incremento en la concentración de metionina en la dieta ha sido documentado por Wang et al. (2010) y Chen et al. (2011), quienes encontraron un aumento en el aporte de este aminoácido metabolizable al utilizar HMBi (ácido 2-hidroxi-4-metilmercapto butírico); por lo que, la utilización de análogos de metionina se considera una opción válida para incrementar su concentración en dietas con cantidades deficientes en este aminoácido.

\section{Conclusiones}

Adicionar HMTBA en dietas deficientes en metionina, cuya fuente proteica proviene de la harina de soya y forraje kikuyo (Kikuyochloa clandestinum), permitió incrementar el aporte de este aminoácido como porcentaje de la PM. Sin embargo, el adicionar HMTBA a las dietas de vacas en pastoreo no produjo una respuesta significativa $(\mathrm{P}>0,05)$ en la producción de leche, leche corregida al 4\% de grasa, ni en los componentes lácteos. Alimentar las vacas con alta concentración de PC incrementó significativamente $(\mathrm{P}<0,05)$ la concentración de NUL de 18,33 a $20,70 \mathrm{mg} / \mathrm{dl}$. No se encontró una retribución económica ante el uso de HMTBA, debido a que no se incrementó la cantidad de leche ni sólidos totales vendibles.

\section{Agradecimientos}

Los autores desean expresar un profundo agradecimiento al Sr. Mario Blanco, propietario de la explotación donde se llevó a cabo el ensayo. 


\section{Literatura citada}

AOAC (Association of Official Analytical Chemists). 1990. Official methods of analysis. 15 ${ }^{\text {th }}$ ed. AOAC, Arlington, VA, USA.

Bartolomeo, M.P., and F. Maisano. 2006. Validation of a reversed-phase HPLC method for quantitative amino acid analysis. J. Biomol. Tech. 17:131-137.

Bremmer, D.R., T.R. Overton, and J.H. Clark. 1997. Production and composition of milk from Jersey cows administered bovine somatotropin and fed ruminally protected amino acids. J. Dairy Sci. 80:1374-80.

Chen, Z.H., G.A. Broderick, N.D. Luchini, B.K. Sloan, and E. Devillard. 2011. Effect of feeding different sources of rumenprotected methionine on milk production and N-utilization in lactating dairy cows. J. Dairy Sci. 94:1978-88.

Elizondo, J. 2006. El nitrógeno en los sistemas ganaderos de leche. Agron. Mesoam. 17:69-77.

Giallongo, F., M. Harper, J. Lopes, H. Lapierre, R. Patton, C. Parys, I. Shinzato, and N. Hristov. 2016. Effects of rumenprotected methionine, lysine, and histidine on lactation performance of dairy cows. J. Dairy Sci. 99:4437-4452.

Hargraves, J.N.G., and J.D. Kerr. 1978. Botanal: a comprehensive sampling and computing procedure for estimating pasture yield and composition. II. Computational package. Commonwealth Scientific and Industrial Research Organization (CSIRO). Brisbane, AUS.

Hansen, W.P., D.E. Otterby, J.G. Linn, and J.D. Donker. 1991. Influence of forage type, ratio of forage to concentrate, and methionine hydroxy analog on performance of dairy cows. J. Dairy Sci. 74:1361-1369.

Hristov, A., and F. Giallongo. 2014. Feeding protein to dairy cows- What should be our target? In: M.L. Eastridge, editor, Proc. Tri-State Dairy Nutr. Conf., Fort Wayne. Ohio State University, Columbus, IN, USA. p. 75-84.

Jiménez, J., y J. Elizondo. 2014. Balance de nitrógeno en fincas para la producción de leche en Costa Rica. Agron. Mesoam. 25:151-160.

Jonker, J.S., R.A. Kohn, and R.A. Erdman. 1998. Using milk urea nitrogen to predict nitrogen excretion and utilization efficiency in lactating dairy cows. J. Dairy Sci. 81:2681-2692.

Johnson-Vanwieringen, L.M., J.H. Harrison, D. Davidson, M.L. Swift, M.A. Von-Keyserlingk, M. Vázquez-Anón, and W. Chalupa. 2007. Effects of rumen-undegradable protein sources and supplemental 2-hydroxy-4-(methylthio)-butanoic acid and lysine- $\mathrm{HCl}$ on lactation performance in dairy cows. J. Dairy Sci. 90:5176-5188.

Klangnok, P., P. Lounglawann, and W. Suksombat. 2011. Effects of met hydroxy analog (MHA $\left.{ }^{\circledR}\right)$ supplementation of dairy cow’s diets on milk yield and milk composition. Suranaree J. Sci. Technol. 18:99-108.

Lapierre, H., M. Vázquez-Anón, D. Parker, P. Dubreuil, G. Holtrop, and G.E. Lobley. 2011. Metabolism of 2-hydroxy-4(methylthio) butanoate (HMTBA) in lactating dairy cows. J. Dairy Sci. 94:1526-35.

Leonardi, C., M. Stevenson, and L.E. Armentano. 2003. Effect of two levels of crude protein and methionine supplementation on performance of dairy cows. J. Dairy Sci. 86:4033-4042.

NRC (National Research Council). 2001. Nutrient requirements of dairy cattle. $7^{\text {th }}$ ed. National Academy Press, WA, USA.

Patterson, J., and L. Kung. 1988. Metabolism of DL-methionine and methionine analogs by rumen microorganisms. J. Dairy Sci. 71:3292-3301.

Piepenbrink, M.S., T.R. Overton, and J.H. Clark. 1996. Response of cows fed a low crude protein diet to ruminally protected methionine and lysine. J. Dairy Sci. 79:1638-1646. 
Phipps, R.H., C.K. Reynolds, D.I. Givens, A.K. Jones, P. Geraert, E. Devillard, and R. Bennett. 2008. Short communication: effects of 2-hydroxy-4-(methylthio) butanoic acid isopropyl ester on milk production and composition of lactating Holstein dairy cows. J. Dairy Sci. 91:4002-4005.

Polan, C.E., K.A. Cummins, C.J. Sniffen, T.V. Muscato, J.L. Vicini, B.A. Crooker, and S.B. Peirce-Sandner. 1991. Responses of dairy cows to supplemental rumen-protected forms of methionine and lysine. J. Dairy Sci. 74:2997-3013.

Rulquin, H., and L. Delaby. 1997. Effects of the energy balance of dairy cows on lactational responses to rumen-protected methionine. J. Dairy Sci. 80:2513-2522.

Rulquin, H., B. Graulet, L. Delaby, and J.C. Robert. 2006. Effect of different forms of methionine on lactational performance of dairy cows. J. Dairy Sci. 89:4387-4394.

SAS. 2004. SAS/STAT 9.1 User's guide. Version 9.1 ed. SAS Institute Inc., Cary, NC, USA.

Schwab, C.G., C.K. Bozak, N.L. Whitehouse, and M.M. Mesbah. 1992. Amino acid limitation and flow to duodenum at four stages of lactation. 1. Sequence of lysine and methionine limitation. J. Dairy Sci. 75:3486-3502.

Schwab, C.G. 1995. Rumen protected amino acids - their role in nutrition of high producing ruminants. In: M. Ivan, editor, Animal science research and development: moving toward a new century. Can. Soc. Anim. Sci., Ottawa, ON, CAN. p. $161-175$

Sharma, B.K., and R.A. Erdman.1988. Abomasal infusion of choline and methionine with or without 2-amino-2-methyl-1propanol for lactating dairy cows. J. Dairy Sci. 71:2406-2411.

St-Pierre, N.R., and J.T. Sylvester. 2005. Effects of 2-hydroxy-4-(methylthio) butanoic acid (HMB) and its isopropyl ester on milk production and composition by Holstein cows. J. Dairy Sci. 88:2487-2497.

Van-Soest, P.J., J.B. Robertson, and B.A. Lewis. 1991. Methods for Dairy fiber, neutral detergent fiber, and nonstarch polysaccharides in relation to animal nutrition. J. Dairy Sci. 74:3583-3597.

Wang, C., H.Y. Liu, Y.M. Wang, Z.W. Yang, J.X. Liu, Y.M. Wu, and H.W. Ye. 2010. Effects of dietary supplementation of methionine and lysine on milk production and nitrogen utilization in dairy cows. J. Dairy Sci. 93:3661-3670.

Whelan, S.J., F.J. Mulligan, B. Flynn, C. McCarney, and K.M. Pierce. 2011. Effect of forage source and a supplementary methionine hydroxy analog on nitrogen balance in lactating dairy cows offered a low crude protein diet. J. Dairy Sci. 94:5080-5089.

Wittcoff, H., B. Reuben, and J.S. Plotkin. 2013. Industrial organic chemicals. $3^{\text {th }}$ ed. Wiley, NJ, USA.

Xu, S., J.H. Harrison, W. Chalupa, C. Sniffen, W. Julien, H. Sato, and T. Fujieda. 1998. The effect of ruminal bypass lysine and methionine on milk yield and composition of lactating cows. J. Dairy Sci. 81:1062-1077.

Zanton, G.I., G.R. Bowman, M. Vázquez-Anón, and L.M. Rode. 2014. Meta-analysis of the lactation performance in dairy cows receiving supplemental dietary methionine sources or postruminal infusion of methionine. J. Dairy Sci. 97:1-17. 